\title{
Uniform Decoration of Reduced Graphene Oxide Sheets with Gold Nanoparticles
}

\author{
Huanping Yang, ${ }^{1}$ Weiwei Zhou, ${ }^{1}$ Bo Yu, ${ }^{1}$ Yingying Wang, ${ }^{1}$ Chunxiao Cong, ${ }^{1}$ and Ting Yu ${ }^{1,2}$ \\ ${ }^{1}$ Division of Physics and Applied Physics, School of Physical and Mathematical Sciences, \\ Nanyang Technological University, Singapore 637371 \\ ${ }^{2}$ Department of physics, Faculty of Science, National University of Singapore, Singapore 117542
}

Correspondence should be addressed to Ting Yu, yuting@ntu.edu.sg

Received 11 July 2011; Revised 8 December 2011; Accepted 23 December 2011

Academic Editor: Renu Pasricha

Copyright (C) 2012 Huanping Yang et al. This is an open access article distributed under the Creative Commons Attribution License, which permits unrestricted use, distribution, and reproduction in any medium, provided the original work is properly cited.

A simple method employing ionic liquid functionalization is developed to achieve the uniform decoration of reduced graphene oxide sheets with gold nanoparticles. The synthesis of ionic liquid modified graphene oxide is accomplished by covalently binding 1-(3-Aminopropyl) imidazole with GO sheets. The formation mechanism of Au nanoparticles on RGO sheets is proposed to load $\mathrm{AuCl}_{4}{ }^{-}$onto the surface of GO sheets through anion exchange; then reduce $\mathrm{AuCl}_{4}{ }^{-}$to Au NPs and at the same time reduce GO sheets to RGO sheets via a one-step process. The presence of Au NPs is well identified by SEM, TEM, and XPS. As a concept, the RGO-supported Au NPs is applied to surface-enhanced Raman spectroscopy.

\section{Introduction}

Graphene is a single layer of carbon atoms that are densely packed into hexagonal structure. It exhibits extraordinary electronic, thermal, and mechanical properties, which make it to be a promising candidate for a variety of applications [1]. Graphene oxide originated from the oxidative exfoliation of graphite, as one of the graphene derivatives, is solutiondispersible due to the oxygen functional groups bearing on the basal planes and edges. From the chemical point of view, the presence of oxygen functionalities on the surface of GO must be very interesting because they can serve as active sites for chemical modification. Therefore, GO can be considered as promising nanoscale building blocks with ultrahigh surface area to generate new nanocomposites.

Recently, accompanying with the emergence of various graphene oxide/reduced graphene oxide (GO/RGO)-based composites or hybrid materials, different kinds of methods have been developed to realize such an integration of GO/ RGO with other materials like polymers, quantum dots, and metal/metal oxide nanoparticles (NPs) [2-11]. For example, $\mathrm{Fu}$ et al. reported that by using in situ synthesis method they synthesized the graphene-metal nanoparticle based derivatives, which give the surface-enhanced Raman scattering properties (SERS) [12]. Zhu et al. construct a hybrid three-dimensional (3D) nanocomposite film by alternatively assembling the ionic liquid (IL) and Pt nanoparticles (PtNPs) modified graphene nanosheets [13].

However, room temperature ionic liquids (RTILs), an efficient system with all the advantages of homogeneous and heterogeneous catalysis, is rarely used for preparing GO/RGO-NPs composites [13]. Herein, we present a simple method to fabricate the RGO-Au nanocomposites, by using ionic liquid as a linker [14]. After realizing the functionalization of GO with ionic liquid 1-(3-Aminopropyl) imidazole, uniform distribution of gold NPs are readily accomplished on the surface of RGO sheets by reducing GO and the $\mathrm{AuCl}_{4}{ }^{-}$anions pre attached on GO via anion exchange. Comparing with other work [15-20], through using the ionic liquids we selectively functionalize the carboxyl group with the ionic liquids. Simultaneously, the Au nanoparticle will uniformly covalent bond with GO. Finally, we get the water soluble RGO-Au nanocomposite, which can be used as a good SERS substrate. This facile approach to synthesis nanocomposites will contribute to the synthesis of GO/RGObased nanocomposites. 

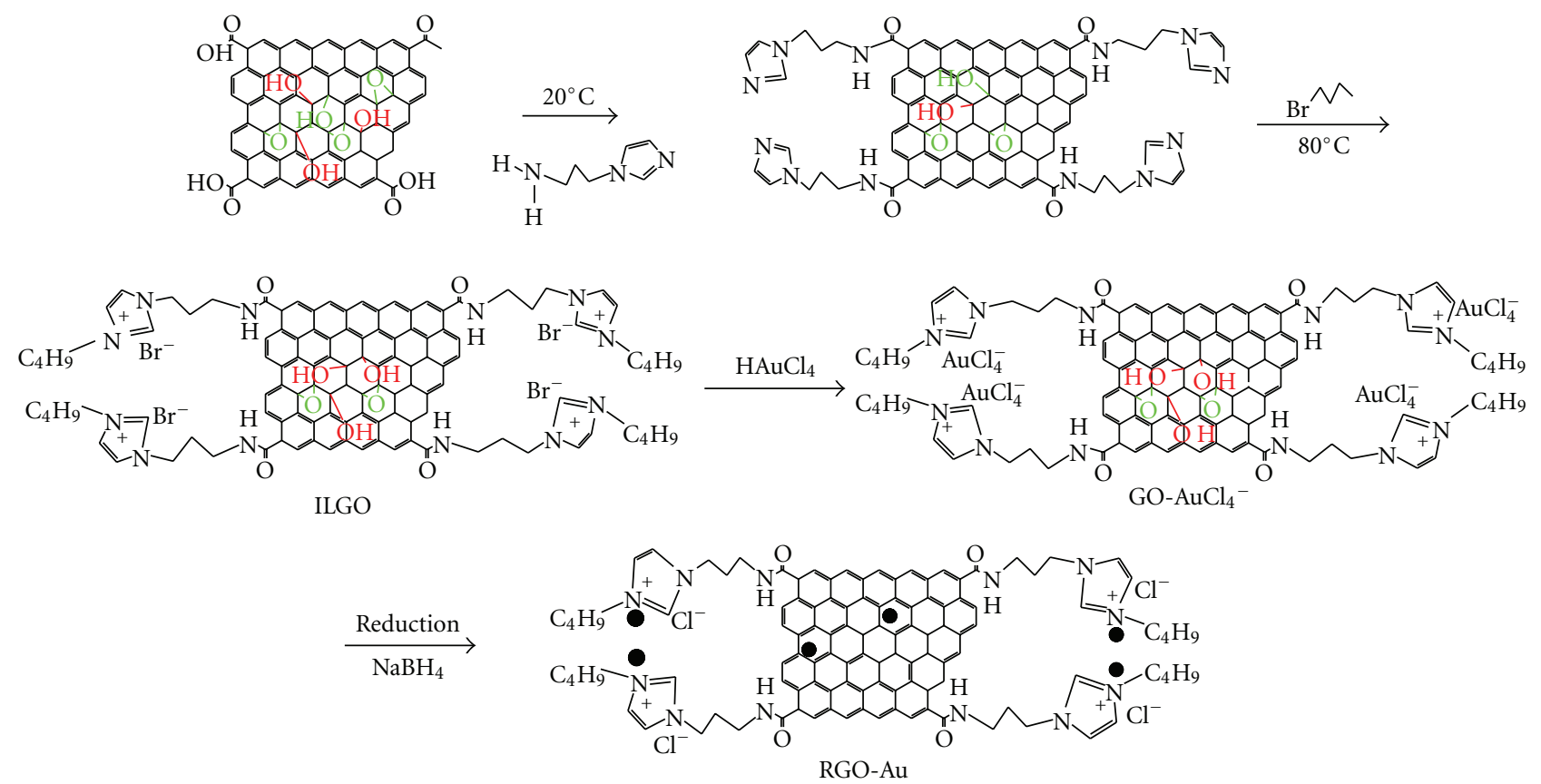

Scheme 1: The formation mechanism for RGO-Au nanocomposites. ${ }^{\bullet}$ Au nanoparticles.

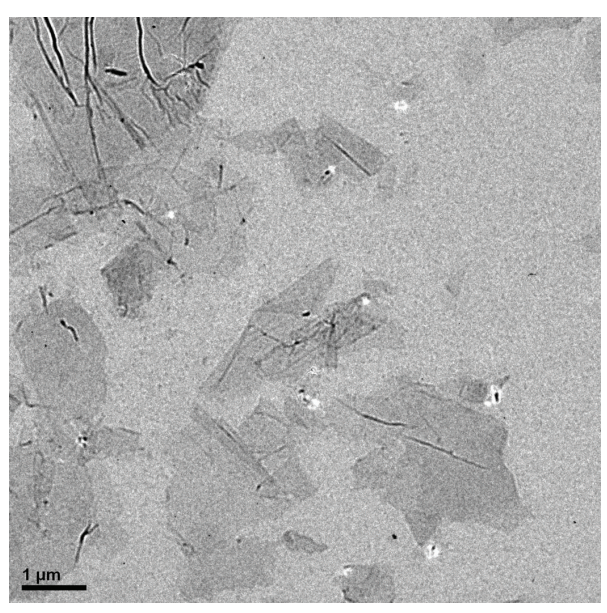

(a)

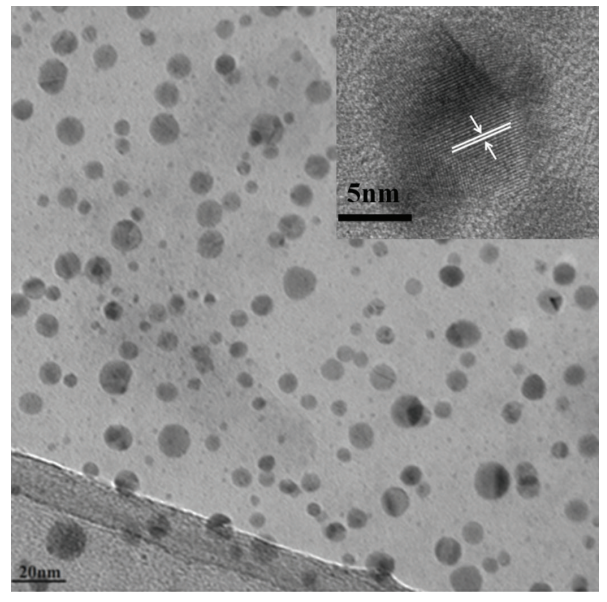

(b)

Figure 1: (a) TEM image of bare GO; (b) TEM and HRTEM image of RGO-Au.

\section{Experiment}

2.1. Materials. Natural graphite,1-(3-Aminopropyl) imidazole, $98 \% \mathrm{H}_{2} \mathrm{SO}_{4}, 30 \% \mathrm{H}_{2} \mathrm{O}_{2}$, potassium permanganate $\left(\mathrm{KMnO}_{4}\right)$, anhydrous Tetrahydrofuran (THF), N, N'-dicyclohexylcarbodiimide (DCC), Rhodamine 6G (R6G), Chloroauric acid $\left(\mathrm{HAuCl}_{4}, 99.9 \%\right), \mathrm{NaNO}_{3}, \mathrm{NaBH}_{4}$, and 1bromobutane were purchased from Sigma-Aldrich and used as received. Double distilled water was used in all the processes of aqueous solution preparation and washing.

2.2. Measurements. The field emission-scanning electron microscopy (FE-SEM) images and distribution of typical elements were obtained on a (JEOL JSM-6700F) field-emission electron microscope with an energy-dispersive X-ray analyzer (EDXA) attachment. Transmission electron microscopy (TEM) analyses were conducted on a (JEM 2010F) electron microscope operated at $120 \mathrm{kV}$. The Raman spectra were recorded using a WITEC-CRM200 Raman system. The excitation source is $532 \mathrm{~nm}$ laser $(2.33 \mathrm{eV})$ with laser power below $0.1 \mathrm{~mW}$ to avoid laser-induced heating. X-ray photoelectron spectroscopy (XPS) measurement, was carried out on a thermo scientific ESCALAB 250.

2.3. Synthesis of Graphene Oxide (GO). GO was synthesized by a modified Hummer's method [21-23]. In brief, $2 \mathrm{~g}$ 
graphite and $1.5 \mathrm{~g} \mathrm{NaNO}_{3}$ were added into $150 \mathrm{~mL} 98 \%$ $\mathrm{H}_{2} \mathrm{SO}_{4}$ solution in a flask which was immersed in an ice bath. $9 \mathrm{~g} \mathrm{KMnO}_{4}$ was then slowly added into the solution, at the same time the temperature of the mixed solution was maintained below $20^{\circ} \mathrm{C}$ for $2 \mathrm{~h}$ to avoid overheating and explosion. After the mixture was stirred for 5 days, $10 \mathrm{~mL} 30 \% \mathrm{H}_{2} \mathrm{O}_{2}$ was added into the solution in order to completely react with the remaining $\mathrm{KMnO}_{4}$, and a bright yellow solution was obtained. Finally, the resulting mixture was washed by $3 \% \mathrm{H}_{2} \mathrm{SO}_{4}$ and then $\mathrm{H}_{2} \mathrm{O}$ until the $\mathrm{pH}$ of the solution was $5 \sim 6$. GO powder was obtained after freeze drying of the suspension.

\subsection{Preparation of Ionic Liquid Functionalized GO (ILGO).} At first, GO sheets and 1-(3-Aminopropyl) imidazole were dispersed into anhydrous THF with sonication for 20 minutes. Subsequently, the DCC solution was slowly added into the mixture, and the above mixture under nitrogen atmosphere was stirred for 6 hours. Then, 1-bromobutane was added into the above mixture. Finally, the homogeneous solution was vigorously stirred at $80^{\circ} \mathrm{C}$ under nitrogen atmosphere for 24 hours. The resulting chemically converted GO sheets (labeled as ILGO) was subsequently centrifuged, washed with water, and air-dried.

2.5. Process of Anion Exchange (GO-AuCl$\left.{ }_{4}^{-}\right)$. At first, ionic liquid functionalized GO was dispersed in DI water and followed by sonication for 20 minutes, Then, aqueous $\mathrm{HAuCl}_{4}$ solution was subsequently added into the above solution. Subsequently, the mixture was being strongly stirred for 24 hours. Finally, DI water was used to wash the sample for several times in order to remove the residual $\mathrm{AuCl}_{4}{ }^{-}$ions. The anion exchange mixture was labeled as $\mathrm{GO}-\mathrm{AuCl}_{4}{ }^{-}$.

2.6. Preparation of the Composite (RGO-Au). The $\mathrm{AuCl}_{4}{ }^{-}$ ions and GO sheets were reduced by $\mathrm{NaBH}_{4}$ in this step. $\mathrm{AuCl}_{4}{ }^{-}$ions were reduced to gold nanoparticles. GO sheets were also reduced to RGO. The RGO acted as the supporter to stabilize the gold nanoparticles. The nanocomposites were labeled as RGO-Au.

\section{Results and Discussions}

On the basis of our experimental procedure, the mechanism to interpret the formation of the as-obtained RGO-Au nanocomposites is put forward, as shown in Scheme 1. As reported previously [24], GO sheets have carbonyl groups on their basal planes and at the edges. These functional groups, act as anchor sites, covalently bind with the ionic liquids (ILGO), which enable the subsequent anion exchange. Then, the $\mathrm{AuCl}_{4}{ }^{-}$ions attached on the surfaces and edges of GO sheets $\left(\mathrm{GO}-\mathrm{AuCl}_{4}{ }^{-}\right.$) [25]. The RGO-Au nanocomposites (RGO-Au) are synthesized, through one-step reduction.

The morphology of the as-prepared RGO-Au nanocomposites were studied by transmission electron microscopy (TEM) and high-resolution transmission electron microscopy (HRTEM). Figure 1(a) reveals that GO sheets are few layers with dimension of a few micrometers. Figure $1(\mathrm{~b})$ is

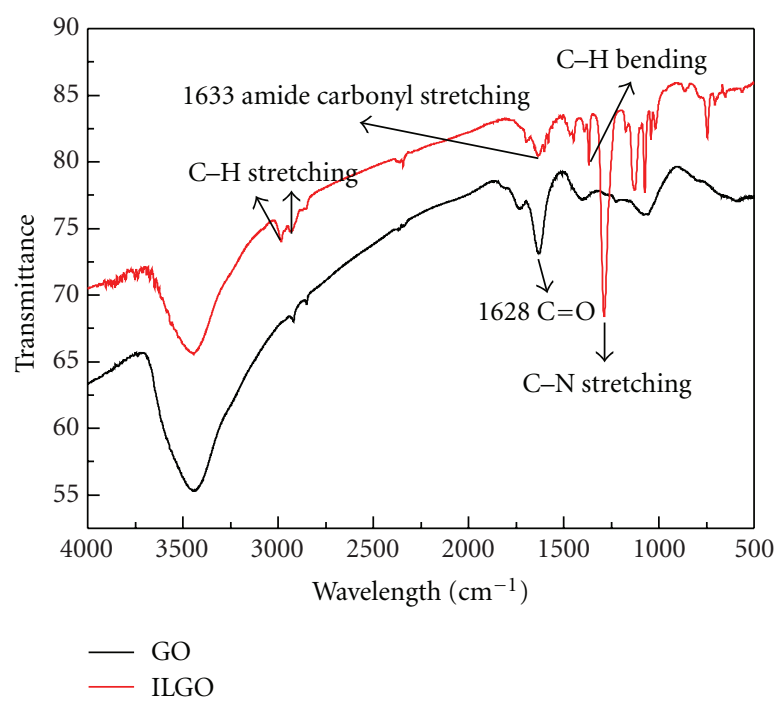

FIgURE 2: FTIR of GO (black) and ILGO (red).

a representative TEM image of the as-synthesized RGO-Au nanocomposites (RGO-Au). Au NPs on RGO surface with uniform dispersion can be clearly seen, the integrity of carbon skeleton has been kept after the chemical process meanwhile. The inserted image shows the HRTEM of the Au nanoparticles. The lattice spacing measured was $0.24 \mathrm{~nm}$, which was close to the lattice spacing, $0.236 \mathrm{~nm}$, for (111) bulk metal face-centered cubic (fcc) Au crystals [26].

Fourier transform-infrared spectroscopy (FTIR) was performed to characterize the GO and IL functional GO nanosheets, shown in Figure 2. Similar with other people's work $[14,27,28]$, the new stretch at $1288 \mathrm{~cm}^{-1}$ originates from $\mathrm{C}-\mathrm{N}$ stretching mode in amido bond. Simultaneously, the new band at $1633 \mathrm{~cm}^{-1}$ in the ILGO can be assigned to the amide carbonyl stretching mode. Moreover, the doublet [14] at $2849 \mathrm{~cm}^{-1}$ and $2944 \mathrm{~cm}^{-1}$ corresponds to symmetric $v_{\text {as }}\left(\mathrm{CH}_{2}\right)$ and asymmetric $v_{\text {as }}\left(\mathrm{CH}_{2}\right)$ of the IL units. The existences of the doublet and the $\mathrm{C}-\mathrm{H}$ bending modes of the ILGO demonstrate that the IL units have been grafted on the GO sheets basal plane. All these indicate that the IL is chemically attached to the GO sheets.

The C1s XPS spectra of GO and RGO-Au are shown in Figures 3(a) and 3(b). They all show the broad of the peak than that of graphite [29], indicating that there are some higher binding energy signals corresponding to $\mathrm{C}-\mathrm{O}$ species [30, 31]. So we use three prominent peaks to fit the $\mathrm{C} 1 \mathrm{~s}$ spectrum, which can be assigned to $\mathrm{sp}^{2}-\mathrm{C}(\sim 285 \mathrm{eV})$, $\mathrm{C}-\mathrm{O}$ (hydroxyl and epoxy, $\sim 287 \mathrm{eV}$ ), and $\mathrm{C}=\mathrm{O}$ (carboxyl, $\sim 289 \mathrm{eV})$, respectively $[24,32,33]$. The C1s spectrum of GO indicates the carbon species are highly oxidized. However, for RGO-Au nanocomposites, the decrease of $\mathrm{C}=\mathrm{O}$ and $\mathrm{C}-$ $\mathrm{OH}$ binding energy at $285 \sim 288 \mathrm{eV}$ implies the successful removal of exogenous functional groups by the chemical reduction. XPS measurement was carried out to further investigate the formation of Au NPs on the IL-modified GO sheets. Figure 3(c) presents the XPS peaks for $\mathrm{Au}^{3+}$ in the form of a doublet at $87.3\left(4 \mathrm{f}_{7 / 2}\right)$ and $90.3 \mathrm{eV}\left(4 \mathrm{f}_{5 / 2}\right)$ [34], 


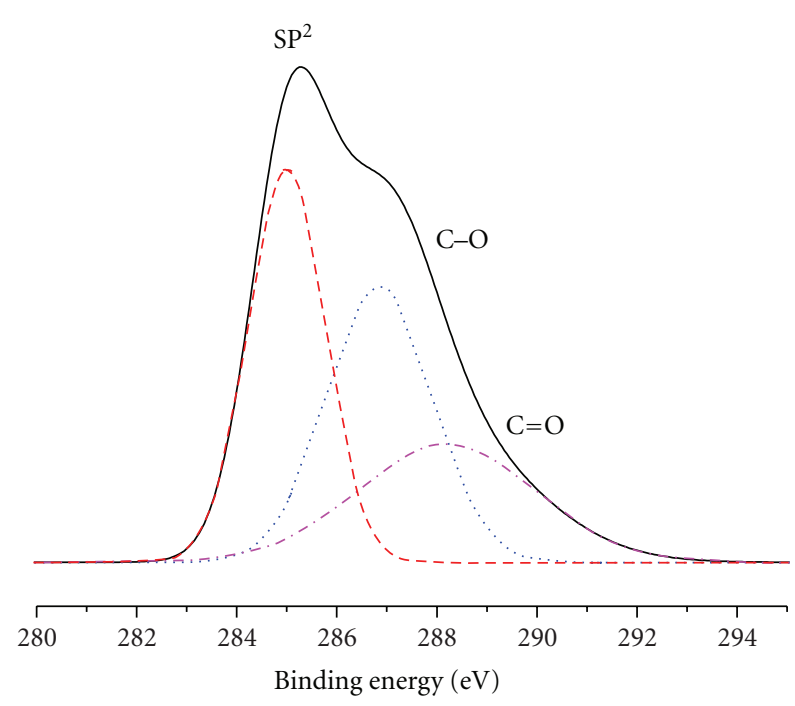

(a)

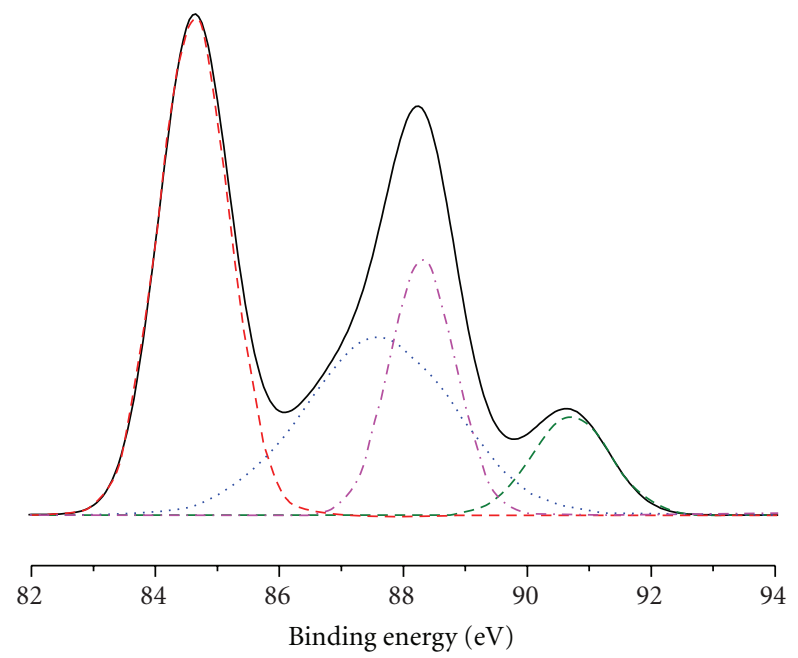

(c)

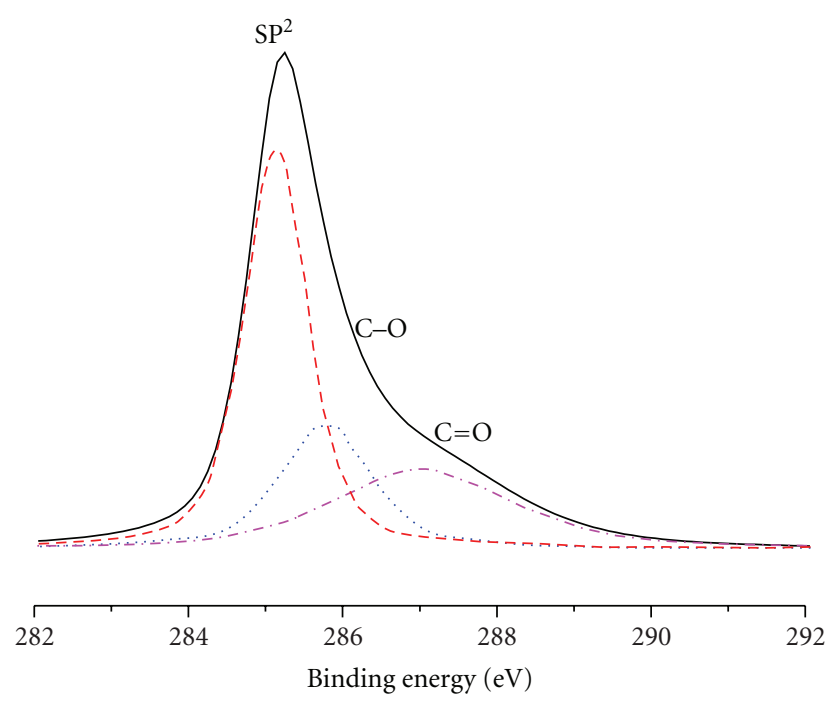

(b)

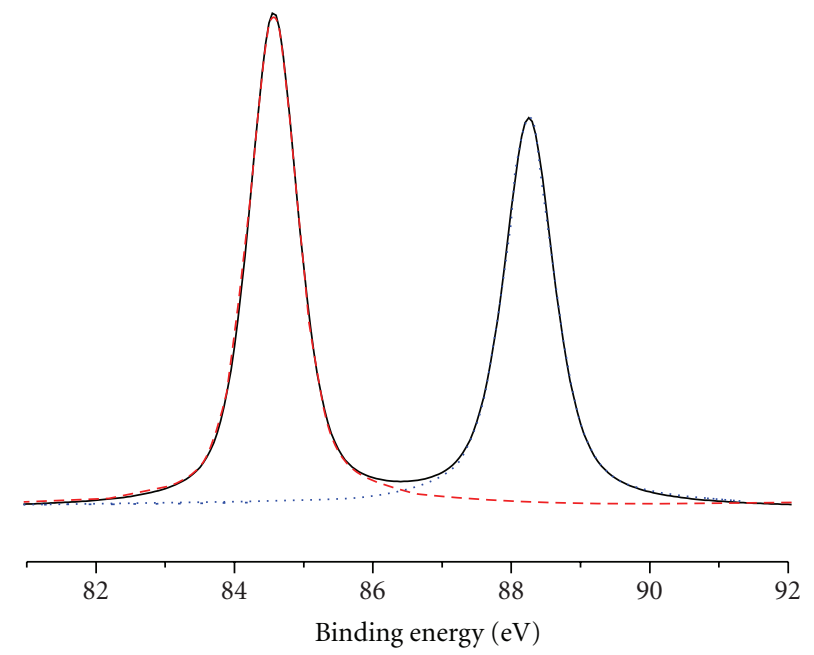

(d)

FIgURE 3: C1s XPS spectra of GO (a) and RGO-Au (b). The black solid lines are original spectra; the dash line, dot line and dash, and dot line are assigned to $\mathrm{sp}^{2}-\mathrm{C}, \mathrm{C}-\mathrm{O}$ (hydroxyl and epoxy groups), $\mathrm{C}=\mathrm{O}$ (carboxyl groups), respectively. Au4f XPS spectra for the $\mathrm{GO}-\mathrm{AuCl}_{4}{ }^{-}$ and RGO-Au nanocomposites: (c) GO- $\mathrm{AuCl}_{4}{ }^{-}$and (d) $\mathrm{RGO}-\mathrm{Au}$.

indicating that $\mathrm{Au}^{3+}$ species exist in the $\mathrm{GO}$ composites. Figure 3(d) shows the doublet peak Au4f $\mathrm{f}_{7 / 2}$ and Au4f $5 / 2$ with binding energies of 84.3 and $88.0 \mathrm{eV}$, respectively. These are typical values for $\mathrm{Au}^{0}$ [35], revealing the formation of gold NPs on the RGO sheets. After reduction, the disappearance of two peaks at 87.3 and $90.3 \mathrm{eV}$ (Figure 3(d)) manifests that no $\mathrm{Au}^{3+}$ specie exists in the RGO composites. It can be seen from the above analysis of the XPS data that Au NPs are insitu loaded onto the functionalized RGO.

Figure 4 gives the N1s XPS spectra for the RGO-Au nanocomposites. It can be clearly seen that the RGO-Au nanocomposites show two prominent peaks, indicating that the amide bonding and the pyrrole-type nitrogen-containing functional groups [36, 37]. Successful grafting of the ionic liquid molecule on the GO sheets can be further deduced.

Energy dispersive X-ray analysis (EDXA) data of RGO$\mathrm{Au}$ shows that the RGO sheets are decorated with $\mathrm{Au}$ nanoparticles. Figures 5(a) and 5(b) show the SEM and quantitative analysis of RGO-Au. It is performed with the $\mathrm{Au}$ (M) lines in the EDX spectrum. The Au content is estimated to be $10-20 \%$ in all the areas. Figures $5(\mathrm{c})$ and $5(\mathrm{~d})$ show the EDXA plane distributions of the typical elements on the surface of RGO-Au, which further confirm the presence of elements $\mathrm{C}$ and $\mathrm{Au}$.

Figure 6 shows the Raman spectra of GO, ILGO, GO$\mathrm{AuCl}_{4}{ }^{-}$, and RGO-Au, which reveal two prominent peaks: D band $\left(1346 \mathrm{~cm}^{-1}\right)$ and $\mathrm{G}$ band $\left(1597 \mathrm{~cm}^{-1}\right)$. It clearly shows that the $G$ and $D$ peak are broader than that of graphite [38]. As we know higher disorder in graphite leads to a broader $G$ band as well as to a broad $D$ band of higher relative intensity compared to that of the $G$ band [38]. D band originates from a double resonance (DR) Raman process involving iTO phonon and defects, while $\mathrm{G}$ band originates from in-plane vibration of $\mathrm{sp}^{2}$ carbon atoms, which is a double degenerate 


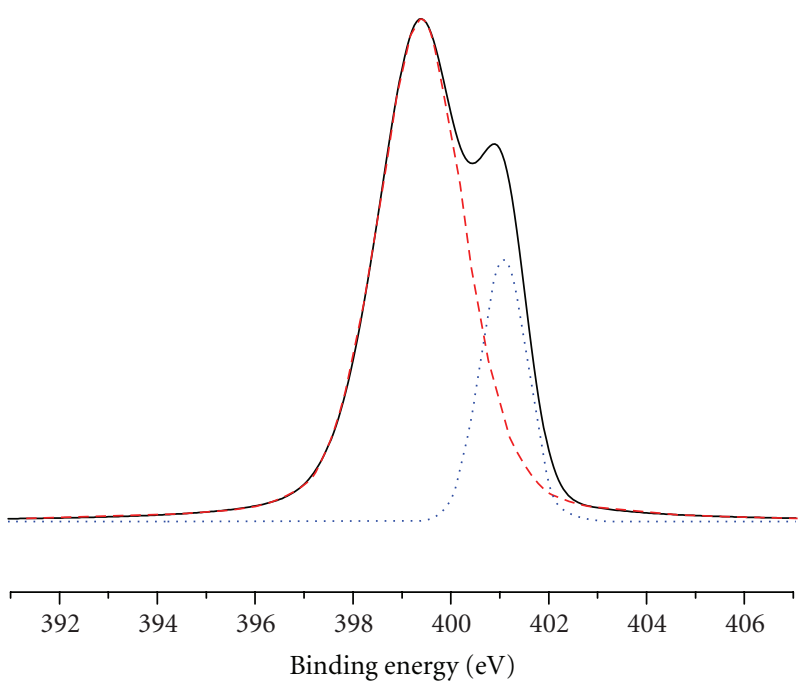

FIGURE 4: N1s XPS spectra for the RGO-Au nanocomposites.
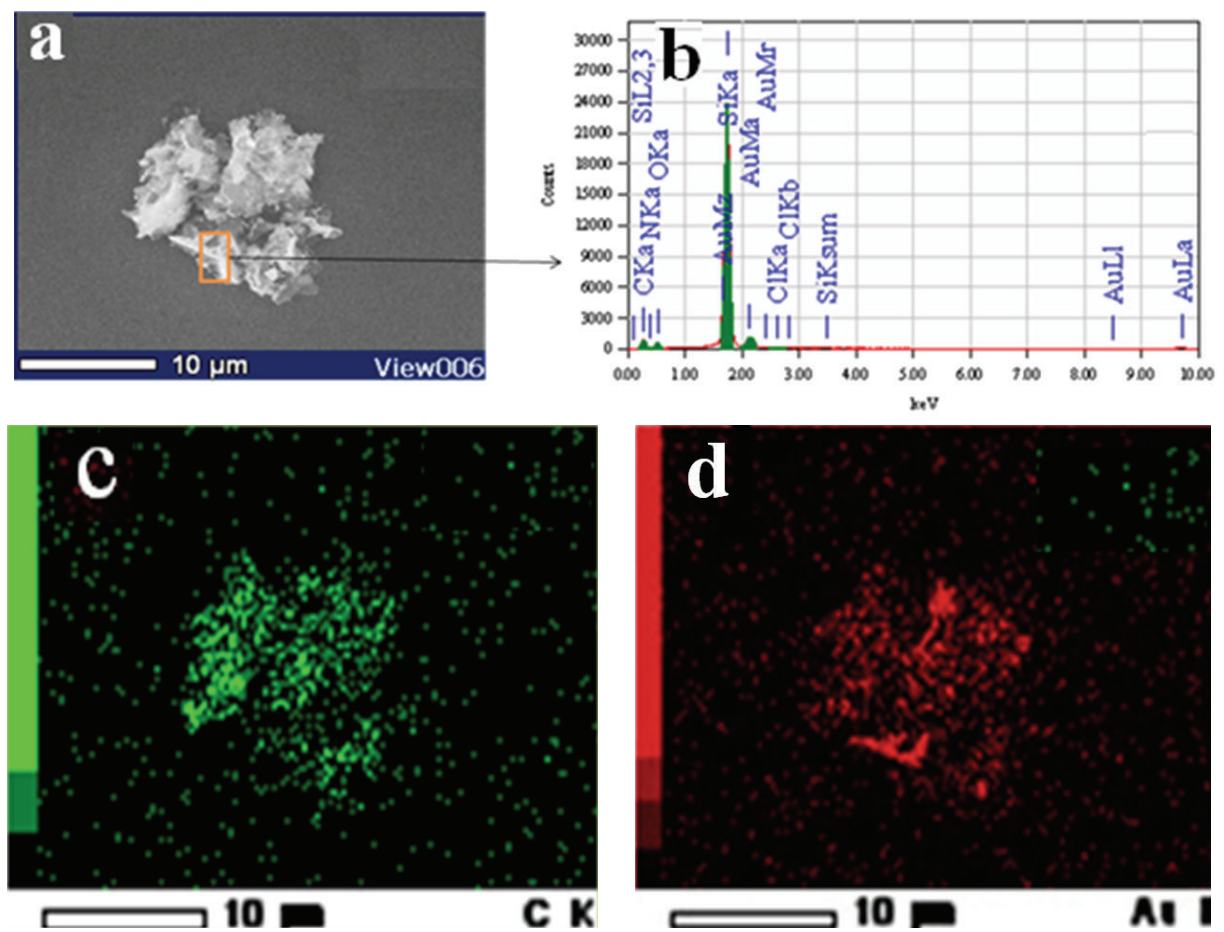

Figure 5: (a) SEM image and (b) results of the EDX element analysis of RGO-Au. (c) EDXA-C and (d) EDXA-Au mapping image of sample RGO-Au. The bright spots show the distribution of $\mathrm{C}$ and Au elements on the RGO-Au surface.

(iTO and LO) phonon mode ( $\mathrm{E}_{2 \mathrm{~g}}$ symmetry) at the Brillouin zone center $(\Gamma$ point $)$. It can be seen from Figure 6 that after functionalization of GO with ionic liquid, anion exchange, and reduction occur, demonstrated by the increased of the intensity ratio of $\mathrm{D}$ to $\mathrm{G}$ band $\left(I_{\mathrm{D}} / I_{\mathrm{G}}\right)$ of GO in Table 1 . The $\mathrm{G}$ band position of RGO-Au shows red shift compared with that of GO, indicating the relaxation of compressive strain in pristine GO sheet. Moreover, the G band of RGO presents more prominent due to the restoring $\mathrm{sp}^{2}$ carbon regions after the chemical reduction treatment [39]. The increased
$I_{\mathrm{D}} / I_{\mathrm{G}}$ ratio is believed to be caused by the decrease of the mean crystallite size of GO. After removal of the exogenous functional groups in the GO sheets by chemical reduction, and the size of the reestablished GO network is smaller than the original one, which leads to an increase in the $I_{D} / I_{G}$ ratio $[24,32,40]$.

RGO-supported Au NPs can be used as a substrate for surface-enhanced Raman spectroscopy (SERS). Rhodamine 6G (R6G) molecules, which are commonly probe molecules in SERS experiments, are used to test the efficiency of this 


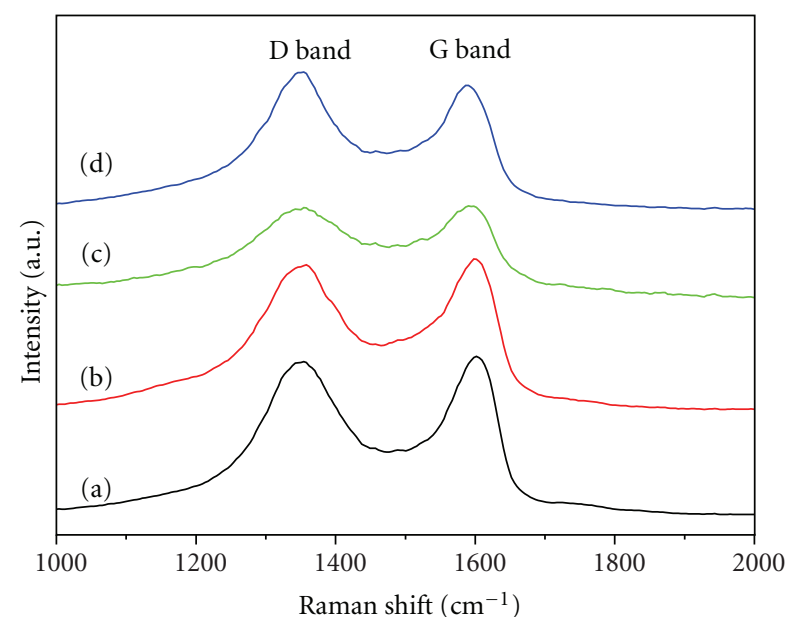

Figure 6: Raman spectrum of (a) GO, (b) ILGO, (c) GO-AuCl ${ }^{-}$, and (d) RGO-Au.

TABLE 1: The position of $\mathrm{G}$ band and values of $I_{\mathrm{D}} / I_{\mathrm{G}}$ obtained from Raman spectra.

\begin{tabular}{lcc}
\hline Samples & G band position $\left(\mathrm{cm}^{-1}\right)$ & $I_{\mathrm{D}} / I_{\mathrm{G}}$ \\
\hline $\mathrm{GO}$ & 1597.2 & 1.6 \\
$\mathrm{ILGO}$ & 1592.5 & 1.9 \\
$\mathrm{GO}^{-A u C l}{ }_{4}^{-}$ & 1590.4 & 2.0 \\
$\mathrm{RGO}-\mathrm{Au}$ & 1587.6 & 2.2 \\
\hline
\end{tabular}

kind of substrate. The RGO with Au NPs was first soaked in a $10^{-6} \mathrm{M}$ R6G solution for about $20 \mathrm{~min}$, washed with distilled water to remove those unabsorbed molecules, and dried in air. For the $\mathrm{R} 6 \mathrm{G}$ on $\mathrm{SiO}_{2} / \mathrm{Si}$ substrate, we dropped $10^{-6} \mathrm{M}$ R6G on $\mathrm{SiO}_{2} / \mathrm{Si}$ substrate and let it dry overnight. Figure 7 shows the Raman spectra of the $\mathrm{R} 6 \mathrm{G}$ on $\mathrm{SiO}_{2} / \mathrm{Si}$ and RGO-supported Au NPs substrate. No Raman signal of the R6G molecules is detected on the $\mathrm{SiO}_{2} / \mathrm{Si}$, due to the low concentration of R6G $\left(10^{-6} \mathrm{M}\right)$. However, for the same concentration of R6G, the Raman signals on RGO-supported $\mathrm{Au}$ NPs substrate are much stronger. In our previous paper [41], we reported that Au/SLG is a much better substrate than Au film alone: the former gives SERS enhancement of the molecules and can efficiently quench the PL background from both the Au and the molecules. Yu et al. also reported that GO can notably enhance the graphene-metal/molecule binding, which will study the impacts of local chemical groups and global $\Pi$-conjugation network on the SERS of molecules [42]. So, we can say that our RGO-supported Au NPs is a promising SERS substrate. The assignment of Raman bands of R6G can be found in [43]. Several spectrums which are taken from different positions on RGO-supported $\mathrm{Au}$ NPs substrate present similar behavior.

From Figure 7, it also can be seen that the fluorescence backgrounds of $\mathrm{R} 6 \mathrm{G}$ on $\mathrm{SiO}_{2} / \mathrm{Si}$ and RGO-supported $\mathrm{Au}$ NPs substrate are significantly different. The relative weak fluorescence background of R6G on RGO-supported Au NPs substrate is due to the resonance energy transfer from R6G to GO [44]. The relative strong Raman signal and weak

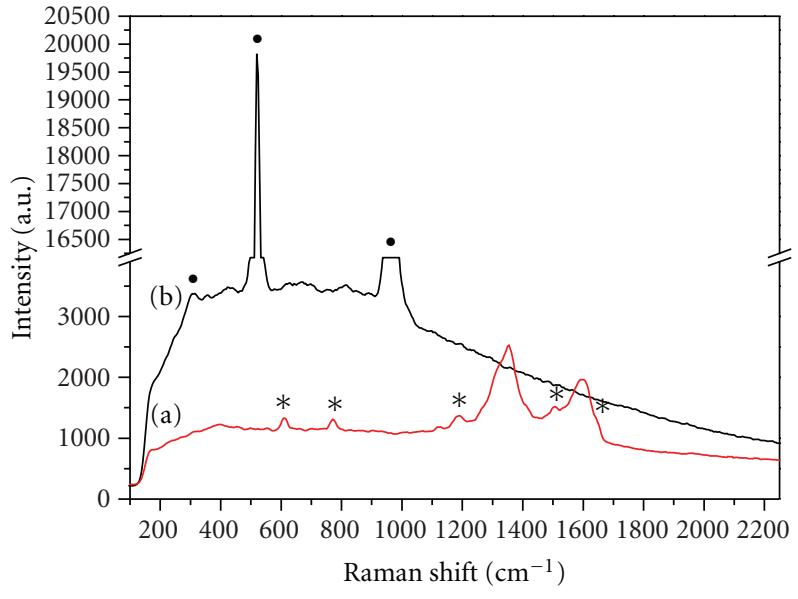

Figure 7: Raman spectrum of the R6G on (a) RGO-Au and (b) $\mathrm{SiO}_{2} / \mathrm{Si}$ substrate. The peaks marked by the stars $\left(^{*}\right)$ and the circle dots $(\bullet)$ are the peaks of R6G and Si, respectively.

molecule fluorescence background make RGO-supported Au NPs a promising SERS substrate.

\section{Conclusions}

In conclusion, by prefunctionalizing GO with imidazolium cation-based ionic liquids, we successfully synthesize RGO sheet with uniformly distributed Au NPS with size less than $10 \mathrm{~nm}$. The formations of Au NPs on RGO sheet are characterized by TEM, XPS, Raman, and EDXA. The reduction product, RGO-supported Au NPs substrate, shows good SERS effect, as proved by using R6G as a probe molecule. This method provides a simple way for preparing the RGOnoble metals or RGO-metal oxide nanocomposites, such as RGO-Pt, RGO-Ag, RGO-ZnO, and GO- $\mathrm{MnO}_{2}$, which can be used for biosensor, catalysis, and SERS applications.

\section{References}

[1] K. S. Novoselov, A. K. Geim, S. V. Morozov et al., "Electric field in atomically thin carbon films," Science, vol. 306, no. 5696, pp. 666-669, 2004.

[2] Y. Li and Y. Wu, "Coassembly of graphene oxide and nanowires for large-area nanowire alignment," Journal of the American Chemical Society, vol. 131, no. 16, pp. 5851-5857, 2009.

[3] H. Kim, S. W. Kim, Y. U. Park et al., " $\mathrm{SnO}_{2}$ /graphene composite with high lithium storage capability for lithium rechargeable batteries," Nano Research, vol. 3, no. 11, pp. 813-821, 2011.

[4] P. Wang, T. Jiang, C. Zhu, Y. Zhai, D. Wang, and S. Dong, "One-step, solvothermal synthesis of graphene-CdS and graphene-ZnS quantum dot nanocomposites and their interesting photovoltaic properties," Nano Research, vol. 3, no. 11, pp. 794-799, 2010.

[5] Z. S. Wu, W. Ren, D. W. Wang, F. Li, B. Liu, and H. M. Cheng, "High-energy $\mathrm{MnO} 2$ nanowire/graphene and graphene asymmetric electrochemical capacitors," ACS Nano, vol. 4, no. 10, pp. 5835-5842, 2010. 
[6] X. Zhao, Q. Zhang, Y. Hao, Y. Li, Y. Fang, and D. Chen, "Alternate multilayer films of poly(vinyl alcohol) and exfoliated graphene oxide fabricated via a facial layer-by-layer assembly," Macromolecules, vol. 43, no. 22, pp. 9411-9416, 2010.

[7] H. Wang, H. S. Casalongue, Y. Liang, and H. Dai, "Ni(OH)2 nanoplates grown on graphene as advanced electrochemical pseudocapacitor materials," Journal of the American Chemical Society, vol. 132, no. 21, pp. 7472-7477, 2010.

[8] Q. Wu, Y. Xu, Z. Yao, A. Liu, and G. Shi, "Supercapacitors based on flexible graphene/polyaniline nanofiber composite films," ACS Nano, vol. 4, no. 4, pp. 1963-1970, 2010.

[9] S. Chen, J. Zhu, X. Wu, Q. Han, and X. Wang, "Graphene oxide-Mno2 nanocomposites for supercapacitors," ACS Nano, vol. 4, no. 5, pp. 2822-2830, 2010.

[10] X. Zhou, X. Huang, X. Qi et al., "In situ synthesis of metal nanoparticles on single-layer graphene oxide and reduced graphene oxide surfaces," Journal of Physical Chemistry C, vol. 113, no. 25, pp. 10842-10846, 2009.

[11] G. Goncalves, P. A. A. P. Marques, C. M. Granadeiro, H. I. S. Nogueira, M. K. Singh, and J. Grácio, "Surface modification of graphene nanosheets with gold nanoparticles: the role of oxygen moieties at graphene surface on gold nucleation and growth," Chemistry of Materials, vol. 21, no. 20, pp. 47964802, 2009.

[12] X. Fu, F. Bei, X. Wang, S. O'Brien, and J. R. Lombardi, "Excitation profile of surface-enhanced Raman scattering in graphene-metal nanoparticle based derivatives," Nanoscale, vol. 2, no. 8, pp. 1461-1466, 2010.

[13] C. Zhu, S. Guo, Y. Zhai, and S. Dong, "Layer-by-layer selfassembly for constructing a graphene/platinum nanoparticle three-dimensional hybrid nanostructure using ionic liquid as a linker," Langmuir, vol. 26, no. 10, pp. 7614-7618, 2010.

[14] H. Yang, C. Shan, F. Li, D. Han, Q. Zhang, and L. Niu, "Covalent functionalization of polydisperse chemically-converted graphene sheets with amine-terminated ionic liquid," Chemical Communications, no. 26, pp. 3880-3882, 2009.

[15] G. Lu, H. Li, C. Liusman, Z. Yin, S. Wu, and H. Zhang, "Surface enhanced Raman scattering of Ag or Au nanoparticledecorated reduced graphene oxide for detection of aromatic molecules," Chemical Science, vol. 2, no. 9, pp. 1817-1821, 2011.

[16] T. A. Pham, B. C. Choi, K. T. Lim, and Y. T. Jeong, "A simple approach for immobilization of gold nanoparticles on graphene oxide sheets by covalent bonding," Applied Surface Science, vol. 257, no. 8, pp. 3350-3357, 2011.

[17] Y. Zhang, S. Liu, W. Lu, L. Wang, J. Tian, and X. Sun, "In situ green synthesis of Au nanostructures on graphene oxide and their application for catalytic reduction of 4-nitrophenol," Catalysis Science and Technology, vol. 1, no. 7, pp. 1142-1144, 2011.

[18] S. Zhang, Y. Shao, H.-G. Liao et al., "Graphene decorated with PtAu alloy nanoparticles: facile synthesis and promising application for formic acid oxidation," Chemistry of Materials, vol. 23, no. 5, pp. 1079-1081, 2011.

[19] N. Zhang, H. Qiu, Y. Liu et al., "Fabrication of gold nanoparticle/graphene oxide nanocomposites and their excellent catalytic performance," Journal of Materials Chemistry, vol. 21, no. 30, pp. 11080-11083, 2011.

[20] K. Gotoh, T. Kinumoto, E. Fujii et al., "Exfoliated graphene sheets decorated with metal/metal oxide nanoparticles: simple preparation from cation exchanged graphite oxide," Carbon, vol. 49, no. 4, pp. 1118-1125, 2011.
[21] W. S. Hummers and R. E. Offeman, "Preparation of graphitic oxide," Journal of the American Chemical Society, vol. 80, no. 6, article 1339, 1958.

[22] Y. Hernandez, V. Nicolosi, M. Lotya et al., "High-yield production of graphene by liquid-phase exfoliation of graphite," Nature Nanotechnology, vol. 3, no. 9, pp. 563-568, 2008.

[23] Y. Xu, H. Bai, G. Lu, C. Li, and G. Shi, "Flexible graphene films via the filtration of water-soluble noncovalent functionalized graphene sheets," Journal of the American Chemical Society, vol. 130, no. 18, pp. 5856-5857, 2008.

[24] S. Stankovich, D. A. Dikin, R. D. Piner et al., "Synthesis of graphene-based nanosheets via chemical reduction of exfoliated graphite oxide," Carbon, vol. 45, no. 7, pp. 1558-1565, 2007.

[25] C. Xu, X. Wang, J. Zhu, X. Yang, and L. Lu, "Deposition of Co3O4 nanoparticles onto exfoliated graphite oxide sheets," Journal of Materials Chemistry, vol. 18, no. 46, pp. 5625-5629, 2008.

[26] G. Xie, M. Song, and K. Furuya, "Fabrication and characterization of Au-nanoparticle/W-nanodendrite structures on $\mathrm{Al}_{2} \mathrm{O}_{3}$ substrate," Journal of Materials Science, vol. 41, no. 14, pp. 4537-4542, 2006.

[27] S. Stankovich, R. D. Piner, S. T. Nguyen, and R. S. Ruoff, "Synthesis and exfoliation of isocyanate-treated graphene oxide nanoplatelets," Carbon, vol. 44, no. 15, pp. 3342-3347, 2006.

[28] X. Zhang, Y. Huang, Y. Wang, Y. Ma, Z. Liu, and Y. Chen, "Synthesis and characterization of a graphene-C60 hybrid material," Carbon, vol. 47, no. 1, pp. 334-337, 2009.

[29] X. Li, G. Zhang, X. Bai et al., "Highly conducting graphene sheets and Langmuir-Blodgett films," Nature Nanotechnology, vol. 3, no. 9, pp. 538-542, 2008.

[30] S. Stankovich, R. D. Piner, X. Chen, N. Wu, S. T. Nguyen, and R. S. Ruoff, "Stable aqueous dispersions of graphitic nanoplatelets via the reduction of exfoliated graphite oxide in the presence of poly(sodium 4-styrenesulfonate)," Journal of Materials Chemistry, vol. 16, no. 2, pp. 155-158, 2006.

[31] C. Hontoria-Lucas, A. J. López-Peinado, J. D. D. LópezGonzález, M. L. Rojas-Cervantes, and R. M. Martín-Aranda, "Study of oxygen-containing groups in a series of graphite oxides: physical and chemical characterization," Carbon, vol. 33, no. 11, pp. 1585-1592, 1995.

[32] W. Guoxiu, Y. Juan, P. Jinsoo et al., "Facile synthesis and characterization of graphene nanosheets," Journal of Physical Chemistry C, vol. 112, no. 22, pp. 8192-8195, 2008.

[33] D. Zhan, Z. Ni, W. Chen et al., "Electronic structure of graphite oxide and thermally reduced graphite oxide," Carbon, vol. 49, no. 4, pp. 1362-1366, 2011.

[34] J. A. Smith, M. Josowicz, and J. Janata, "Polyaniline-gold nanocomposite system," Journal of the Electrochemical Society, vol. 150, no. 8, pp. E384-E388, 2003.

[35] W. Cheng, S. Dong, and E. Wang, "ynthesis and selfassembly of cetyltrimethylammonium bromide-capped gold nanoparticles," Langmuir, vol. 19, no. 22, pp. 9434-9439, 2003.

[36] P. Burchill and L. S. Welch, "Variation of nitrogen content and functionality with rank for some UK bituminous coals," Fuel, vol. 68, no. 1, pp. 100-104, 1989.

[37] R. J. J. Jansen and H. van Bekkum, "XPS of nitrogen-containing functional groups on activated carbon," Carbon, vol. 33, no. 8, pp. 1021-1027, 1995.

[38] K. N. Kudin, B. Ozbas, H. C. Schniepp, R. K. Prud'homme, I. A. Aksay, and R. Car, "Raman spectra of graphite oxide and functionalized graphene sheets," Nano Letters, vol. 8, no. 1, pp. 36-41, 2008.

[39] H. Kang, A. Kulkarni, S. Stankovich, R. S. Ruoff, and S. Baik, "Restoring electrical conductivity of dielectrophoretically 
assembled graphite oxide sheets by thermal and chemical reduc- tion techniques," Carbon, vol. 47, no. 6, pp. 1520-1525, 2009.

[40] Z. Li, J. Zhang, H. He, J. Bian, X. Zhang, and G. Han, "Bluegreen luminescence and SERS study of carbon-rich hydrogenated amorphous silicon carbide films with multiphase structure," Physica Status Solidi (A) Applications and Materials, vol. 207, no. 11, pp. 2543-2548, 2010.

[41] Y. Wang, Z. Ni, H. Hu et al., "Gold on graphene as a substrate for surface enhanced Raman scattering study," Applied Physics Letters, vol. 97, no. 16, Article ID 163111, 3 pages, 2010.

[42] X. Yu, H. Cai, W. Zhang et al., "Tuning chemical enhancement of SERS by controlling the chemical reduction of graphene oxide nanosheets," ACS Nano, vol. 5, no. 2, pp. 952-958, 2011.

[43] P. Hildebrandt and M. Stockhurger, "Surface-Enhanced Resonance Raman Spectroscopy of Rhodamine 6G adsorbed on colloidal silver," Journal of Physical Chemistry, vol. 88, no. 24, pp. 5935-5944, 1984.

[44] X. Ling, L. Xie, Y. Fang et al., "Can graphene be used as a substrate for Raman enhancement?" Nano Letters, vol. 10, no. 2, pp. 553-561, 2010. 

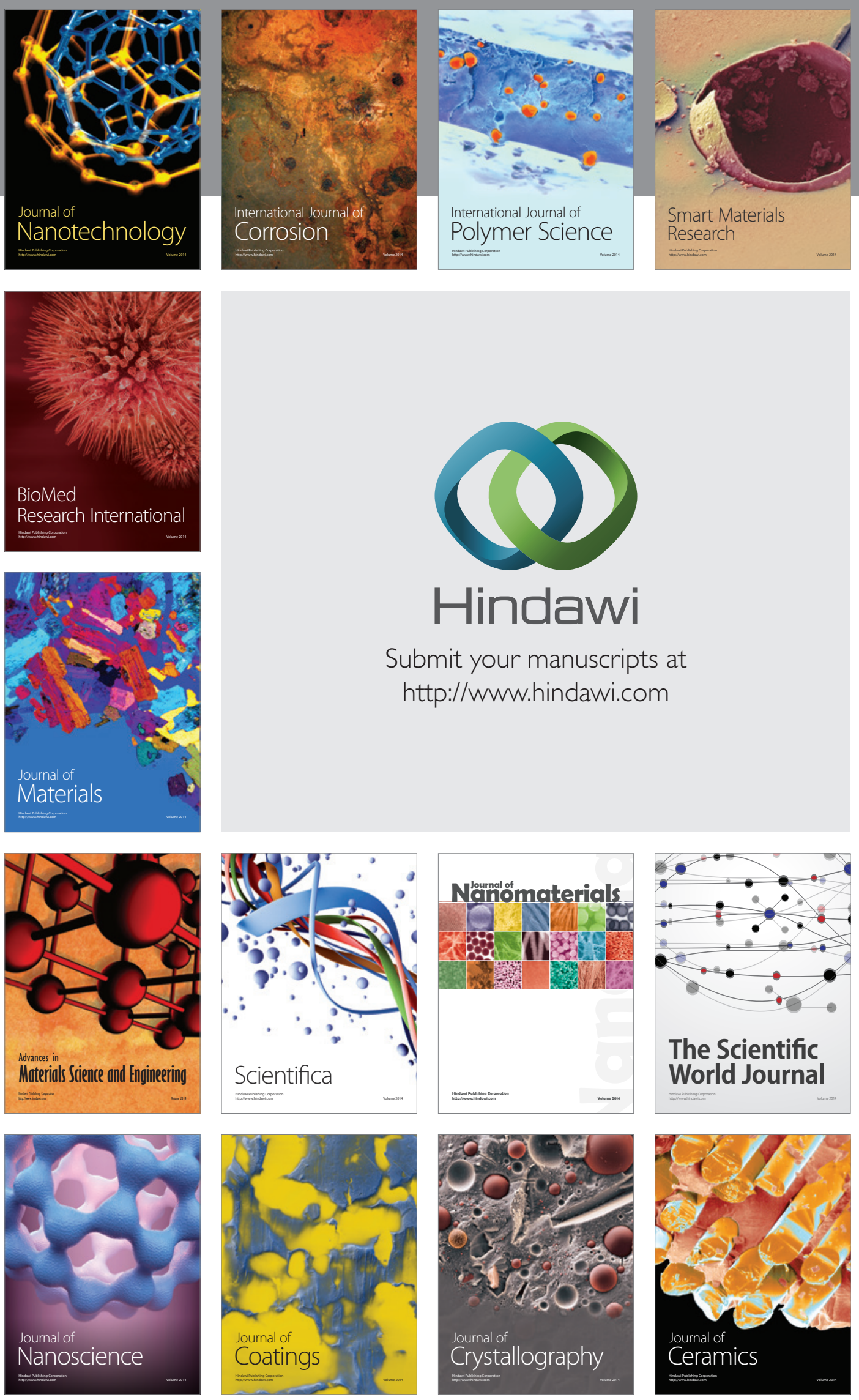

The Scientific World Journal

Submit your manuscripts at

http://www.hindawi.com

\section{World Journal}

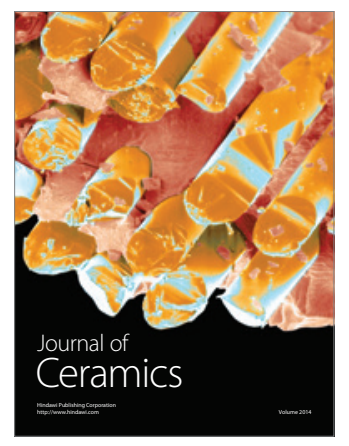

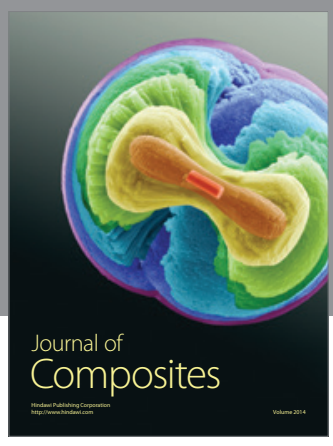
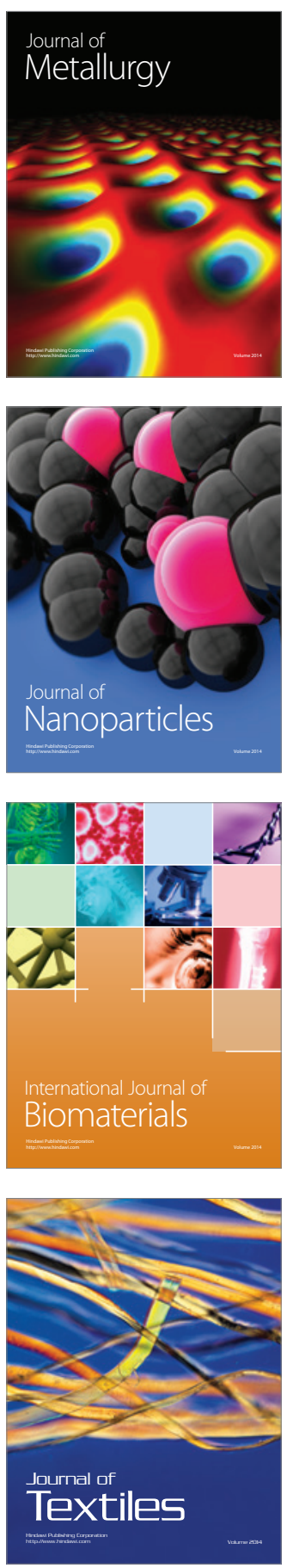